\title{
MS06-02 | Structural Basis Of Ribosomal RNA Synthesis In BACTERIA
}

Wahl, Markus (Freie Universität Berlin, Berlin, GER)

Ribosomal RNA synthesis in Escherichia coli involves a transcription elongation complex (EC), in which RNA polymerase is modified by a signal element on the transcript, Nus factors A, B, E and G, ribosomal protein S4 and inositol mono-phosphatase SuhB. This EC is resistant to $\rho$-dependent termination and facilitates ribosomal RNA folding, maturation and subunit assembly. I will report on recent results from our lab on the functional architecture of the ribosomal RNA EC. 